\title{
REVIEW ARTICLE The NLRP6 inflammasome in health and disease
}

\author{
Laxman Ghimire ${ }^{1}$, Sagar Paudel ${ }^{1}$, Liliang $\operatorname{Jin}^{1}$ and Samithamby Jeyaseelan ${ }^{1,2}$
}

NACHT, LRR (leucine-rich repeat), and PYD (pyrin domain) domain-containing 6 (NIrp6) is a member of the NLR (nucleotideoligomerization domain-like receptor) family that patrols the cytosolic compartment of cells to detect pathogen- and damageassociated molecular patterns. Because Nlrp6 is a recently discovered inflammasome, details of its signaling mechanism, structural assembly, and roles in host defense have yet to be determined. To date, Nlrp6 has been proposed to perform a multitude of functions ranging from control of microbiota, maintenance of epithelial integrity, and regulation of metabolic diseases to modulation of host defense during microbial infections, cancer protection, and regulation of neuroinflammation. While recent studies have questioned some of the proposed functions of Nlrp6, Nlrp6 has been shown to form an inflammasome complex and cleaves interleukin-1 $\beta$ (IL-1 $\beta$ ) and IL-18 during microbial infection, indicating that it is a bonafide inflammasome. In this review, we summarize the recent advancements in knowledge of the signaling mechanisms and structure of the NIrp6 inflammasome and discuss the relevance of NLRP6 to human disease.

Mucosal Immunology (2020) 13:388-398; https://doi.org/10.1038/s41385-020-0256-z

\section{NOD-LIKE RECEPTORS IN IMMUNITY}

The mammalian immune system is endowed with the germlineencoded pattern recognition receptors (PRR) that sense both pathogen-associated molecular patterns (PAMPs) and damageassociated molecular patterns (DAMPs) to initiate proinflammatory responses as a first line of defense. PRRs can be classified into two groups based on their cellular localization. The first group includes Toll-like receptors (TLRs) and C-type lectin receptors that surveil the extracellular and endosomal compartments. The second class of PRRs includes retinoic acid-inducible gene-like receptors and nucleotide-oligomerization domain (NOD)-like receptors (NLRs), which survey intracellular compartments for the presence of PAMPs and DAMPs. ${ }^{1}$ Thus far, 23 NLRs have been identified in humans and more than 30 have been identified in mice; ${ }^{2}$ however, only a few of these have been extensively studied. Predominantly expressed in first responder cells like neutrophils, macrophages, and dendritic cells, NLRs are multi-domain protein complexes consisting of a central NOD (NOD or NACHT) flanked by C-terminal leucine-rich repeats (LRRs) for sensing PAMPs and DAMPs, and a variable $\mathrm{N}$-terminal region consisting of either a caspase activation and recruitment domain (CARD), pyrin domain (PYD), or baculovirus inhibitor repeats. ${ }^{2-7}$ Unlike other classes of receptors, NLRs are unique in the sense that many of them can form a supramolecular complex, known as an inflammasome, by recruiting apoptosis-associated speck-like protein (ASC) and caspase- 1 or -11 after sensing PAMPs or DAMPs. The activated caspase converts the inactive forms of interleukin-1 $\beta$ (IL-1 $\beta$ ) and IL-18 into their respective active forms to initiate inflammatory signaling. ${ }^{8,9}$ Based on the phylogenetic structure of the central NACHT domain, NLRs can be classified into three broad categories: NODs, NACHT, LRR, and PYD domains-containing 6 (NLRPs) or NALPs (NLRP1-14), and the IPAF family of NLRs (NLRC4 and NLR family apoptosis inhibitory proteins). ${ }^{3}$ In case of Nlrp1b, the functional degradation of $\mathrm{N}$-terminal region is involved in pathogen recognition.

Because NLRP6 is a relatively new member of the NLR family, its detailed signaling mechanism is yet to be established. During its initial identification, NLRP6 was shown to activate caspase-1, like other NLRs, and nuclear factor-KB (NF-KB), like TLRs. Thus, it is unique in the NLR family as it can perform functions of both NLRs and TLRs. ${ }^{10}$ This notion is supported by other studies proposing a wide scope of physiological functions of Nlrp6 ranging from modulation of the host-microbial interface ${ }^{11}$ and host defense against pathogens ${ }^{12,13}$ to inhibition of carcinogenesis ${ }^{14}$ and neuroinflammation. ${ }^{15}$ However, Nlrp6 has also been shown to negatively regulate NF-KB signaling in mouse model. ${ }^{13}$ In this review, we discuss the recent advancements in understanding of Nlrp6 inflammasome signaling, summarize the diversified roles of this receptor in host defense and inflammation, and cover recent updates regarding its structure, inflammasome assembly, and regulation of microbiota.

\section{NLRP6 ASSEMBLES AN INFLAMMASOME}

The initial report of NLRP6 by Grenier et al. ${ }^{10}$ in 2002, using human cell lines, demonstrated that co-expression of NLRP6 (PYPAF5) and ASC leads to caspase- 1 and NF-KB activation in COS-7L and 293T cells, respectively. Based on this observation, it was assumed that NLRP6 could form an inflammasome like other members of the NLR family, although in vivo evidence was lacking. The in vivo evidence of this was later provided by two different laboratories at the same time in 2011 when they demonstrated that Nlrp6 ${ }^{-1-}$ mice have reduced serum IL-18 levels under steady-state conditions $^{16}$ and after dextran sulfate sodium (DSS)-induced colitis $^{14,16}$ compared to that of wild-type (WT) counterparts. However, IL-1 $\beta$, which is regarded as one of the signature

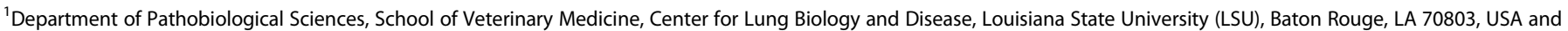
${ }^{2}$ Section of Pulmonary and Critical Care, Department of Medicine, LSU Health Sciences Center, New Orleans, LA 70112, USA
} Correspondence: Samithamby Jeyaseelan (jey@lsu.edu)

Received: 18 August 2019 Revised: 5 January 2020 Accepted: 8 January 2020

Published online: 27 January 2020 
cytokines of inflammasome activation, was found to be upregulated in the colon of Nlrp6 ${ }^{-1-}$ mice. ${ }^{14}$ This may be due to redundant functions of other inflammasome complexes. Nonetheless, Levy et al. ${ }^{17}$ demonstrated that Nlrp6 co-localizes with ASC in intestinal cells under steady-state conditions to form an inflammasome, thus suggesting that Nlrp6 assembles an inflammasome in vivo. However, this finding did not hold true during microbial infection. Anand et al. ${ }^{13}$ reported that they did not observe any obvious difference in the levels of IL-1 $\beta$ or caspase- 1 processing between $\mathrm{NIrp6}^{-1-}$ and WT mice during different bacterial infections, including Salmonella, Listeria, and Escherichia coli. Therefore, at this time, it remained unclear whether Nlrp6 forms an inflammasome during systemic bacterial infections.

Recently, we ${ }^{12}$ and others ${ }^{18}$ reported that the Nlrp6 inflammasome is activated during bacterial infections. In our study, Nlrp6 ${ }^{-}$

bone marrow-derived macrophages (BMDMs) showed reduced caspase-1 processing and IL- $\beta$ secretion compared to that of WT BMDMs when infected with methicillin-resistant Staphylococcus aureus (MRSA). Consistently, MRSA-infected NIrp6 ${ }^{-1-}$ mice had reduced IL-1 $\beta$ secretion in bronchoalveolar lavage fluid compared to that of WT mice. This is strong evidence that Nlrp6 assembles an inflammasome during microbial infection. We observed that the Nlrp3 inflammasome was intact in Nlrp6 ${ }^{-1-}$ BMDMs as treatment with Nlrp3 agonist enhanced IL-1 $\beta$ production in these macrophages. As reported by Grenier et al. ${ }^{10}$ and Levy et al., ${ }^{17}$ we found that NIrp6 co-localizes with ASC in BMDMs after infection. ${ }^{12}$ Similarly, an excellent study from Hara et al. ${ }^{18}$ reported that Grampositive bacteria, such as Listeria monocytogenes, activate the Nlrp6 inflammasome. More importantly, the authors demonstrated that lipoteichoic acid (LTA) from L. monocytogenes upregulates the expression of Nlrp6 and caspase-11 via type 1 interferon (IFN) signaling, which could be regarded as the first signal. As a second signal, LTA serves as a ligand to bind NLRP6 in order to activate the inflammasome via the ASC-caspase-11caspase-1 signaling cascade ${ }^{18}$ (Fig. 1). This growing evidence from independent laboratories suggests that NIrp6 does assemble an inflammasome under both steady-state conditions and during systemic microbial infections. However, further investigations are warranted to determine how S. aureus upregulates Nlrp6 expression in macrophages. Being a Gram-positive bacterium, it is possible that toxins from $S$. aureus could also activate type 1 IFN signaling, as in the case of Listeria, ${ }^{18}$ or regulate TLR signaling, as in the case of Nlrp3, ${ }^{2}$ to upregulate expression of Nlrp6.

\section{MECHANISM OF NLRPG INFLAMMASOME ACTIVATION}

In general, an inflammasome consists of a sensor component, an adapter molecule, and an effector component. The sensor molecule consists of either a PYD or CARD at its $\mathrm{N}$ terminus; the adapter molecule, usually ASC, consists of PYD and CARD domains, and the effector component has a CARD at its end. Based on the sensor component, inflammasomes can be broadly classified into three groups: NLR-associated inflammasomes, absent in melanoma-2 (AIM2)-like receptor (ALR)-associated inflammasomes, and the Pyrin inflammasome. ${ }^{19,20}$ Similarly, based on the effector component, inflammasomes can be canonical (activate caspase-1) or non-canonical (activate caspase-11). All NLR-related inflammasomes are canonical inflammasomes; however, all canonical inflammasomes are not NLR-associated inflammasomes (e.g., AIM2).

Inflammasome autoactivation is thought to be prevented by the closed conformation of the LRR and NACHT domains of NLR (autoinhibition) in the absence of ligand.12 Upon stimulation by ligands, the homotypic interaction between component proteins leads to the assembly of an inflammasome. Biochemical studies have reported prion-like properties of PYD and CARD filaments that facilitate inflammasome assembly. Recently, Cai et al. ${ }^{22}$ and Lu et al. ${ }^{19}$ demonstrated that prion-like polymerization of ASC into filamentous structures is an important step in the activation of ASCdependent inflammasomes. Based on the cryoelectron microscopy structure and structure-based mutagenesis, ASC-dependent inflammasomes were found to share a unified assembly mechanism that
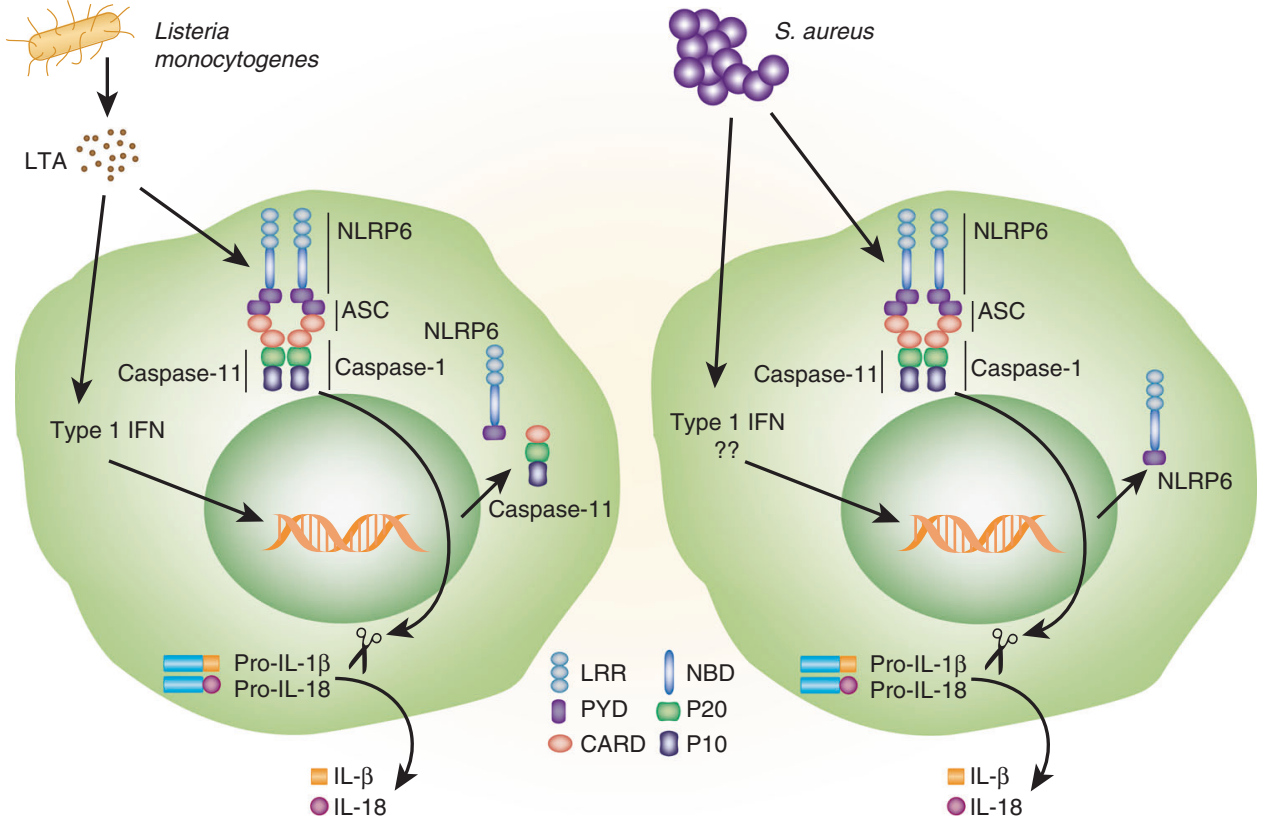

Fig. 1 Activation of the NLRP6 inflammasome after Listeria and S. aureus infections. Cytosolic delivery of Listeria or its toxin (LTA) upregulates the expression of caspase-11 and NLRP6 via triggering of Type 1 IFN signaling in macrophages. NLRP6 then recruits ASC (via PYD-PYD interaction) and caspase-1 and caspase-11 (via CARD-CARD interaction) to form the NLRP6 inflammasome complex. The activated inflammasome cleaves pro-IL-1 $\beta$ and pro-IL-18 into their active forms, which are then secreted from the cell. During S. aureus inflection, NLRP6 is upregulated and recruits ASC and caspase-1, as mentioned above, to form the NRLP6 inflammasome complex. Inactive forms of IL- $1 \beta$ and IL18 are converted into their respective active forms to mediate further immunological responses. PYD: pyrin domain; LRR: leucine-rich repeats; CARD: caspase activation and recruitment domain; LTA: lipoteichoic acid. 
Table 1. Nlrp6 in host defense following microbial infection.

\begin{tabular}{|c|c|c|c|c|c|}
\hline Pathogen & Model used & Phenotype observed & Proposed mechanism of action & $\begin{array}{l}\text { Inflammasome/NF-кB/IFN } \\
\text { dependent }\end{array}$ & Refs. \\
\hline Staphylococcus aureus & Pneumonia (IT) & Nlrp6 $^{-/-}$mice protected & $\begin{array}{l}\text { Nlrp6 augments pyroptosis and } \\
\text { necroptosis of neutrophils and } \\
\text { macrophages, thereby reducing the } \\
\text { number of phagocytes to clear } \\
\text { bacteria. In addition, Nlrp6 deficiency } \\
\text { enhances IFN- } \gamma \text { secretion in the lungs }\end{array}$ & Inflammasome dependent & 12 \\
\hline Listeria monocytogens & Systemic infection (IV) & Nlrp6 $^{-/-}$mice protected & $\begin{array}{l}\text { Nlrp6 inflammasome enhances IL-18 } \\
\text { secretion, which is detrimental } \\
\text { during Listeria infection }\end{array}$ & Inflammasome dependent & 18 \\
\hline Salmonella typhimurium & Systemic infection (IV) & No change in phenotype & & NA & 18 \\
\hline Salmonella typhimurium & Systemic infection (IP) & Nlrp6 ${ }^{-/-}$mice protected & $\begin{array}{l}\text { Nlrp6 deficiency augments NF-кB- } \\
\text { mediated neutrophil recruitment, } \\
\text { which is important to limit } \\
\text { Salmonella }\end{array}$ & $\begin{array}{l}\text { Inflammasome independent, } \\
\text { but NF-кB dependent }\end{array}$ & 13 \\
\hline Escherichia coli & Systemic infection (IP) & Nlrp6 ${ }^{-1-}$ mice protected & $\begin{array}{l}\text { Deletion of Nlrp6 enhances NF-кB- } \\
\text { mediated neutrophil recruitment }\end{array}$ & $\begin{array}{l}\text { Inflammasome independent, } \\
\text { but NF-KB dependent }\end{array}$ & 13 \\
\hline Clostridium rodentium & Enteritis model & Nlrp6 ${ }^{-/-}$mice susceptible & $\begin{array}{l}\text { Nlrp } 6^{-1-} \text { mice had a thin mucus layer } \\
\text { as a result of defective goblet cell } \\
\text { function. This facilitates prolonged } \\
\text { adherence of } C \text {. rodentium }\end{array}$ & Inflammasome dependent & 11 \\
\hline $\begin{array}{l}\text { Encephalomyocarditis } \\
\text { virus (EMCV), Norovirus }\end{array}$ & $\begin{array}{l}\text { Systemic infection (EMCV: } \\
\text { IP and oral; } \\
\text { Norovirus: oral) }\end{array}$ & Nlrp6 ${ }^{-1-}$ mice susceptible & $\begin{array}{l}\text { Nlrp6 recognizes cytosolic long } \\
\text { dsRNA and activated mitochondrial } \\
\text { antiviral signaling proteins (MAVS) to } \\
\text { enhance the antiviral response. } \\
\text { Nlrp6-Dhx15-MAVs axis augments } \\
\text { the expression of interferon- } \\
\text { stimulated genes to limit virus in the } \\
\text { intestine }\end{array}$ & $\begin{array}{l}\text { Inflammasome independent, } \\
\text { but IFN dependent }\end{array}$ & 36 \\
\hline
\end{tabular}

involves two nucleation-induced polymerization steps. ${ }^{19,23}$ The first step is nucleation of ASC PYD filaments by NLR ${ }^{\text {YYD }}$ through a PYD-PYD interaction that leads to polymerization of ASC. In the second step, the polymerized ASC CARD nucleates caspase-1 1 CARD filaments via a CARD-CARD interaction for caspase-1 activation. Activated caspase- 1 is responsible for maturation of pro-IL-1 $\beta$ and pro-IL-18, as well as gasdermin-D-mediated pyroptosis. ${ }^{24}$ Recently, the detailed filamentous and crystal structure of the NLRP6 PYD was reported by Shen et al. ${ }^{25}$ using biochemical and biophysical methods. In addition, the authors have identified the surface that NLRP6 pyrin filament uses to recruit PYD of ASC through molecular dynamics simulations. The authors purified full-length NLRP6 $\left(\mathrm{NLRP6}^{\mathrm{FL}}\right), \mathrm{NLRP6}^{\mathrm{PYD}}$, and NLRP6 ${ }^{\mathrm{PYD}+\mathrm{NBD}}$ through affinity and sizeexclusion chromatography and tested their ability to induce $A S C^{P Y D}$ polymerization. All NLRP6 constructs were able to promote ASC ${ }^{\text {PYD }}$ polymerization. NLRP6 ${ }^{\mathrm{PYD}}$ filament possess a hollow cylindrical structure that assembles through a right-handed helix, and a single NLRP6 ${ }^{\text {PYD }}$ contains six anti-parallel a helices. During activation, most of the conformational changes in NLRP6 occur between the $a 2$ and a3 loop to facilitate interactions.

Compared to AIM2 $2^{\text {PYD }}$, the $a 2-a 3$ loop of NLRP6 ${ }^{\text {PYD }}$ is longer, suggesting that NLRP6 ${ }^{\text {PYD }}$ permits more degrees of conformational change during filament formation than does AIM2 ${ }^{\text {PYD }} \cdot 25,26$ Both NLRP6 and NLRP3 share similar molecular structures with slight differences in the PYD component. However, while NLRP6 $^{\text {PYD }}$ is capable of nucleating ASC ${ }^{\text {PYD }}$ by itself, NLRP3 ${ }^{\text {PYD }}$ is not able to provide a sufficient platform for ASC assembly. ${ }^{19,25}$ Like NLRC4, upon overexpression the full-length NLRP6 assembles into an open and active filamentous conformation with the PYD filament in the center and LRR + NBD outside. However, the LRR + NBD ring was more ordered in NLRC4 than in NLRP6. ${ }^{25,27}$ Since, NLRP6 has been shown to recruit both caspase-1 and caspase-11 during inflammasome assembly, ${ }^{18}$ future biochemical studies are needed to provide additional mechanistic insight into NLRP6 inflammasome assembly.

\section{NLRP6 IN HOST DEFENSE}

Inflammasomes have been shown to regulate host defense mechanisms under different disease conditions. ${ }^{12,28-30}$ The role of the Nlrp3 inflammasome in response to bacterial, viral, and fungal infections has been well established, ${ }^{31-35}$ and there is mounting evidence that Nlrp6 also regulates host defense during different microbial infections (Table 1). An interesting study by Anand et al. ${ }^{13}$ reported that Nlrp6 negatively regulates innate immunity during bacterial infections. In this study, deletion of Nlrp6 was found to enhance NF-KB and mitogen-activated protein kinase (MAPK) activity resulting in increased neutrophil recruitment to the lungs. This augmented neutrophil accumulation was associated with enhanced bacterial clearance and improved survival in Nlrp6 ${ }^{-1-}$ mice infected with Listeria, Salmonella, or E. coli. However, the authors neither depleted neutrophils nor blocked NF-KB or MAPK pathways to confirm this claim. Interestingly, the authors did not find differences in the level of IL-1 $\beta$ or caspase- 1 processing, indicating that the host protection conferred by Nlrp6 deficiency was inflammasome independent. In addition, Nlrp6 regulated bacterial infection independently of microbiota composition as the co-housed Nlrp6 ${ }^{-1-}$ mice demonstrated a reduced bacterial burden compared to that of singly housed mice. However, this study did not elucidate how Nlrp6 recognizes these bacteria to initiate immune signaling cascades.

We recently demonstrated that Nlrp6 serves as a negative regulator of pulmonary host defense during Gram-positive bacterial infection of the lungs. ${ }^{12}$ MRSA has been shown to induce necroptosis in the lungs resulting in pathology; ${ }^{37}$ however, how the necroptosis machinery is regulated in the lungs has remained unclear. We reported that activated Nlrp6 triggers necroptosis and pyroptosis of macrophages and neutrophils in the lungs, which ultimately reduces neutrophil numbers, thereby compromising bacterial clearance (Fig. 2). Through co-housing of Nlrp6 ${ }^{-1-}$ mice with WT mice, we further demonstrated that NIrp6 aggravates MRSA infection independently of microbiota composition. It is interesting that pyroptosis, which was reported to be 

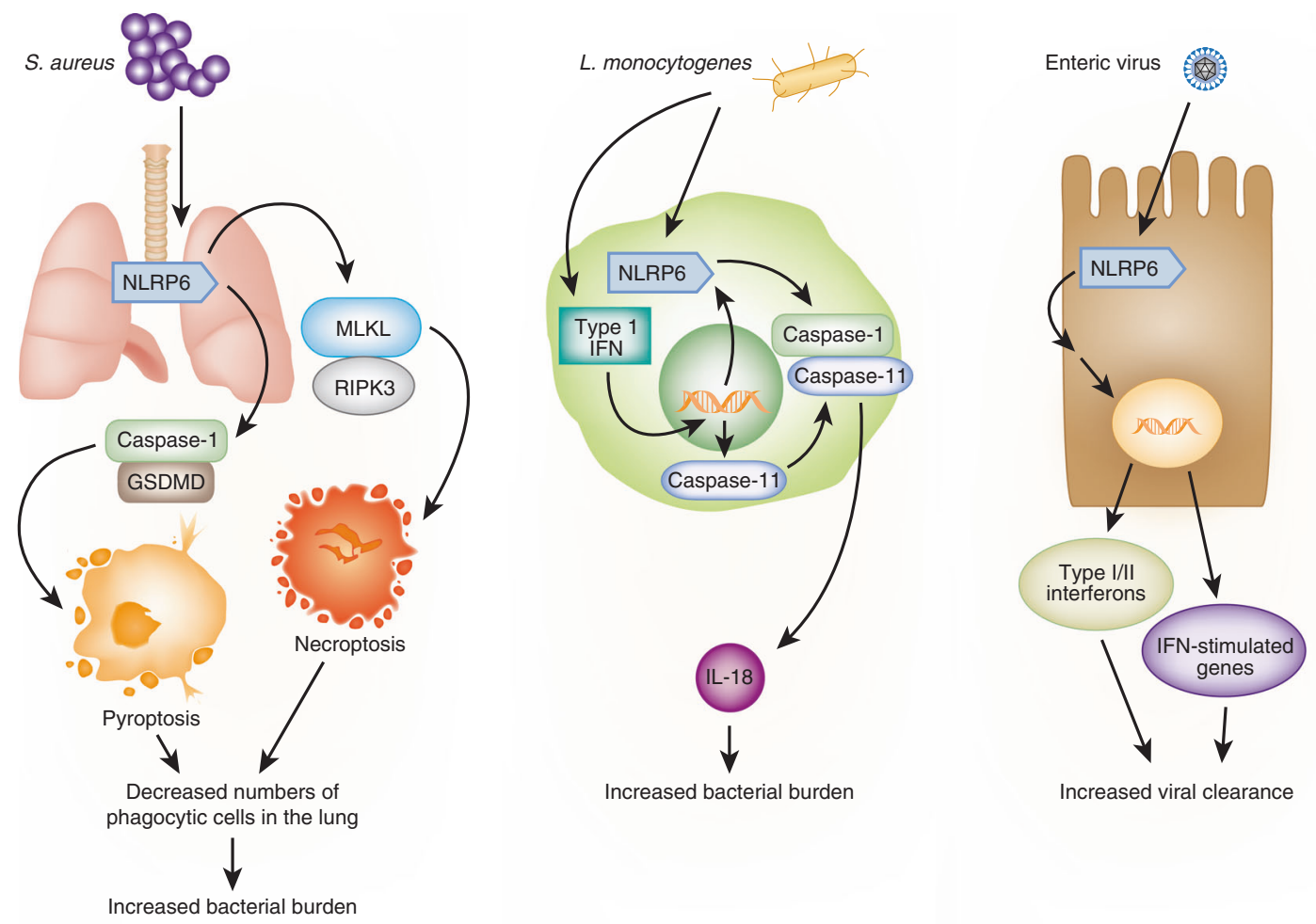

Fig. 2 The NLRP6 inflammasome regulates host protection following microbial infection. a Pulmonary infection with S. aureus activates the NLRP6 inflammasome, which triggers inflammatory modes of cell death, particularly necroptosis and pyroptosis, in the lungs. NLRP6 increases the expression of MLKL and RIPK3 to induce necroptosis and caspase-1 and gasdermin-d (GSDMD) to induce pyroptosis. As a result of increased cell death, the number of phagocytic cells is reduced in the lungs impairing Staphylococcal clearance. $\mathbf{b}$ Systemic infection with Listeria monocytogenes activates the NLRP6-ASC-caspase-1/11 inflammasome complex in macrophages. The activated caspase- 1 cleaves inactive IL-18 into active IL-18, which is then secreted from the cell resulting in increased mortality during Listeria infection. c NLRP6 recognizes cytosolic dsRNA of encephalomyelitis virus via the NLRP6-Dhx15 viral sensing complex in the enterocyte. This complex further activates MAVs to induce the transcription of Type I/III interferons and IFN-stimulated genes for the antiviral response. GSDMD: gasdermin-d; MAVs: mitochondrial antiviral signaling protein; Dhx15: DEAH-box helicase 15.

essential for protection against intracellular pathogens, ${ }^{38}$ was found to be detrimental during pulmonary MRSA infection. These observations indicate that the role of pyroptosis may depend on the lifestyle of a pathogen. For intracellular pathogens, pyroptotic cell death may kill the pathogen along with the cell itself. However, extracellular pathogens might hijack NLRs, such as Nlrp6, NIrc4, ${ }^{28}$ or Nlrp3, ${ }^{32}$ to induce pyroptotic cell death that exacerbates inflammation-induced pathology resulting in detriment to the host. In addition to $S$. aureus-induced pneumonia, it would be revealing to study whether the Nlrp6 inflammasome exerts deleterious or protective effects during Gram-negative bacteria-induced pneumonia, since Gram-negative pathogens have their own lifestyles, virulence factors, and immune evasion strategies.

Recently, an elegant study from Hara et al. ${ }^{18}$ demonstrated that LTA from Listeria binds Nlrp6 and activates the Nlrp6 inflammasome via ASC to regulate host defense during Gram-positive bacterial infection. Interestingly, Nlrp6 activated both caspase-11 and caspase- 1 upon binding of LTA or Listeria for processing of IL$1 \beta$ and IL-18. In contrast, NIrp3, AIM2, and pyrin inflammasomes did not activate caspase-11. Upon infection with Listeria, Nlrp6 ${ }^{-1-}$ and caspase- $11^{-1-}$ mice had reduced bacterial burdens in several organs and better survival compared to that of WT mice, thus confirming that NIrp6 is detrimental during systemic Grampositive pathogen infection. This protection was abolished when these mice received recombinant IL-18, but not IL-1 $\beta$, suggesting that the Nlrp6 inflammasome exacerbates Listeria infection via IL18 production (Fig. 2). In addition, the authors co-housed Nlrp6 ${ }^{-1-}$ mice with WT mice for 4 weeks to determine if the protection observed in Nlrp6 ${ }^{-1-}$ mice is microbiota dependent. Interestingly,
Nlrp6/caspase-11 signaling regulated Listeria infection independently of microbiota. However, future studies will be essential to understand how IL-18 exacerbates Listeria infection as IL-18 has been shown to play protective as well as deleterious roles during such infections. In the same study, the authors also infected WT and Nlrp6 ${ }^{-1-}$ mice with Salmonella and observed bacterial growth in the liver and spleen. In contrast to what was observed in another study using Salmonella, ${ }^{13}$ no differences in bacterial burden were observed between WT and NIrp6 ${ }^{-/-}$mice. ${ }^{13}$ It is difficult to explain this discrepancy in results as both studies used similar bacteria, doses, and similar routes of infection (intravenous (IV) vs. intraperitoneal (IP)). Explanations for this could be the minor difference in application method or differences in tissue sampling as the authors have not clarified whether whole liver or a lobe was used for bacterial load estimation.

Although most studies of microbial infections have presented Nlrp6 as a negative regulator of host defense, some studies have reported essential roles for this molecule. Wlodarska et al. ${ }^{11}$ utilized a murine model of Citrobacter-induced enteritis and found that Nlrp6 deficiency results in impaired host defense. Intestines from NIrp6 ${ }^{-1-}$ mice were extensively colonized with $C$. rodentium and the NIrp6 ${ }^{-1-}$ mice displayed extensive mucosal ulceration, edema, and hyperplasia compared to WT mice. Interestingly, the authors found a thin mucus layer in $\mathrm{NLRP}^{-/-}, \mathrm{ASC}^{-1-}$, and Casp $1 / 11^{-1-}$ mice that appeared to facilitate persistent adherence of $C$. rodentium, thereby increasing the bacterial burden. However, since the authors used non-littermate-controlled animals for the study, these results need to be validated in littermate-controlled animals. Moreover, it is important to note that the authors did not investigate the functions of innate immune cells such as 
macrophages and neutrophils in the clearance of $C$. rodentium. Therefore, whether Nlrp6 regulates the recruitment or functions of these cells during such infections remains unclear.

In addition to bacterial infections, a role for Nlrp6 in viral infections has also been reported. Wang et al. ${ }^{36}$ were the first to demonstrate that Nlrp6 plays a crucial role in limiting viral infections. While WT and NIrp6 ${ }^{-1-}$ mice exhibited no difference in survival when infected IP with encephalomyocarditis virus (EMCV), a +ssRNA virus, the Nlrp6 ${ }^{-1-}$ mice had higher viral loads in the intestine, but not in blood, brain, or heart. This suggests that Nlrp6 may have an important role in viral clearance from the intestine. Interestingly, Nlrp6 ${ }^{-1-}$ mice displayed increased susceptibility to EMCV when it was administered orally, and similar results were obtained for oral infection with murine norovirus. In addition, the authors performed co-housing experiments to determine if this phenotype is dependent on the composition of the gut microbiome in mice and found that this was not the case. As survival after oral administration of EMCV was not affected in $\mathrm{Casp1}^{-1-}$ mice, the authors concluded that NLRP6 regulated antiviral host defense independently of inflammasome via an alternate mechanism. Interestingly, they showed that Nlrp6 interacted with the Dhx15 helicase to form a viral sensing complex that recognized cytosolic long double-stranded DNA (dsRNA) and activated mitochondrial antiviral signaling proteins (MAVS) to initiate the antiviral response. Activation of the Nlrp6-Dhx15-MAVs axis enhanced the expression of IFN-stimulated genes that are essential for limiting virus replication in the intestine (Fig. 2). Further, expression of Nlrp6 in the intestine following viral infection was regulated by type I/III IFNs via the IRF3/7 transcription factors. ${ }^{36}$ Given that Nlrp6 has detrimental roles in Gram-positive bacterial pneumonia, ${ }^{12}$ it would be of interest to investigate whether Nlrp6 exhibits a protective or detrimental role in pulmonary host defense during infection with influenza or rhinoviruses.

Besides its role in host defense during microbial infection, the role of Nlrp6 in gastrointestinal graft-versus-host disease (GVHD) has recently been reported by Toubai et al. ${ }^{39}$ The authors used allogenic hematopoietic cell transplantation (allo-HCT) to investigate whether deletion of Nlrp6 affects the severity of gastrointestinal GVHD following allo-HCT. Intriguingly, Nlrp6 aggravated severity and mortality of gastrointestinal GVHD. Moreover, the phenotype was not dependent on microbiota composition, but dependent on non-hematopoietic cell derived Nlrp6. Future studies are needed to demonstrate if the detrimental effects of Nlrp6 are inflammasome dependent.

While it is clear that Nlrp6 executes essential roles to protect the host against bacterial and viral infections in the intestine, where it is highly expressed, in systemic and pulmonary infections, Nlrp6 expression appears to have detrimental effects. Thus, Nlrp6mediated host defense regulation is likely context dependent. In bacterial infections, where myeloid cells are most important, Nlrp6 seems to trigger destructive inflammation; however, during enteritis, where non-hematopoietic cells (epithelial cells) play crucial roles, the Nlrp6-mediated immune response is protective. Nonetheless, more studies will be necessary to further define the role of Nlrp6 in these systems. Moreover, whether Nlrp6 exerts similar effects in viral, fungal, or Gram-negative bacterial pneumonia and sepsis remains obscure. Defining the roles of Nlrp6 in these infections will allow for better generalization of these conclusions.

\section{NLRP6 AND INTESTINAL MICROBIOTA COMPOSITION}

Nlrp6 is highly expressed in the small and large intestine, especially in enterocytes, colonic goblet cells, and myofibroblasts. ${ }^{14,16,40}$ Hence, most studies on Nlrp6 have concentrated on defining its role in these sites. A pioneering study by Elinav et al. ${ }^{16}$ involving
$16 \mathrm{~S}$ ribosomal RNA analysis of fecal samples from non-littermate $\mathrm{Nlrp6}^{-1-}, \mathrm{ASC}^{-1-}$, and IL-18 ${ }^{-/-}$mice revealed distinct gut microbiota in these mice compared to that of wild-type (WT) mice. For example, there was a higher prevalence of anerobic taxa Prevotellaceae and TM7. These mice were also more susceptible to DSS-induced colitis than were WT mice. This alteration in the microflorae was considered to be colitogenic since transfer of microbiota from Nlrp6 ${ }^{-1-}$, $\mathrm{ASC}^{-/-}$, or IL- $18^{-/-}$mice to WT mice via co-housing for 4 weeks resulted in enhanced susceptibility of WT mice to colitis. Intriguingly, depletion of microbiota using broadspectrum antibiotics reversed this phenomenon. Moreover, reduced IL-18 and increased CCL5 secretion in Nlrp6 ${ }^{-1-}$ mice led to altered microbiota and spontaneous intestinal inflammation. ${ }^{16}$ Based on these observations, Elinav et al. ${ }^{16}$ proposed that Nlrp6 inflammasome regulates gut microbiota composition. In line with this study, Levy et al. ${ }^{17}$ demonstrated that ex-germ-free Nlrp6 ${ }^{-/-}$ mice developed dysbiosis as early as 3 weeks following spontaneous recolonization compared to their ex-germ-free WT counterparts. Since microbiota can influence various physiological conditions ${ }^{41}$ and diseases, this finding opened a new research platform for investigation of the possible roles of the Nlrp6 inflammasome in several physiological conditions, such as colitis, tumorigenesis, and metabolic diseases including non-alcoholic fatty liver disease (NAFLD) and obesity. ${ }^{42-45}$ Nevertheless, it is important to note that both studies used non-littermate control animals, and thus future studies will be necessary to confirm these conclusions.

Non-genetic factors such as differences in animal housing, ${ }^{46}$ familial transmission, ${ }^{47}$ and diet $^{48}$ can modulate microbiota composition, suggesting that littermates are the best control animals to use when investigating the impact of host genetic factors on microbiota regulation. Recently, a study by Robertson et al. ${ }^{49}$ compared co-housing and F2-generation littermate methods for standardization of microbiota between two different sources of mice. Interestingly, the littermate method yielded uniform normalization of microbiota in feces, colon, and ileum. However, extended co-housing was only able to normalize fecal microbiota and the microbial community in the colon and ileum remained stable. Using separately housed, non-littermate WT and Nlrp6 ${ }^{-1-}$ mice, Mamantopoulos et al. ${ }^{50}$ did not observe any overall difference in microbiota composition, which is in contrast with the findings from Elinav et al. ${ }^{16}$ However, Porphyromonadaceae and Bacteroidaceae, but not the Prevotellaceae, bacteria were differentially represented in these mice. Using distance-based redundancy analysis, they found that these differences were due to mother and cage covariates rather than host genetic factors (NIrp6). Furthermore, the authors did not observe obvious differences in microbiome composition when using littermates (Nlrp6 ${ }^{+/+}$) even after lifetime separate housing. ${ }^{50}$ In support of this finding, Lemire et al. ${ }^{51}$ also demonstrated that Nirp6 did not impact gut microbiota composition by using littermate NIrp6 ${ }^{-/-}$and Nlrp6 ${ }^{+/+}$mice, pleading again that Nlrp6 does not regulate microbiota composition. In contrast, Seregin et al. ${ }^{44}$ used NIrp6 $6^{-/-} / \mathrm{IL}-10^{-/-}$and Nlrp6 ${ }^{+/+}$IL- $10^{-/-}$littermate control mice and observed significant differences in microbiota composition between these groups, arguing again that Nlrp6 influences composition of gut microbiota. Since the author used IL-10 $0^{-1-}$ background mice, which have been shown to develop spontaneous colitis, it is difficult to conclude whether the observed difference in microbiota is due to Nlrp6 or IL-10 deficiency-mediated colitis or both. To determine the roles of ASC-dependent inflammasomes in microbiota regulation, Levy et al. ${ }^{45}$ used $\mathrm{Asc}^{-/-}$and $\mathrm{Asc}^{+/+}$littermates and observed differences in microbiota composition, which is in contrast to a study by Mamantopoulos et al. ${ }^{50}$ who performed a similar littermate-controlled experiment. Therefore, it is difficult to explain these contradictory results as both studies used littermate control mice and 16s RNA sequencing to analyze microbiota composition. One interesting study by Galvez et al. ${ }^{52}$ reported that microbiota composition varies greatly within the segregated 
Table 2. Important mouse studies of microbiota-dependent phenotypes.

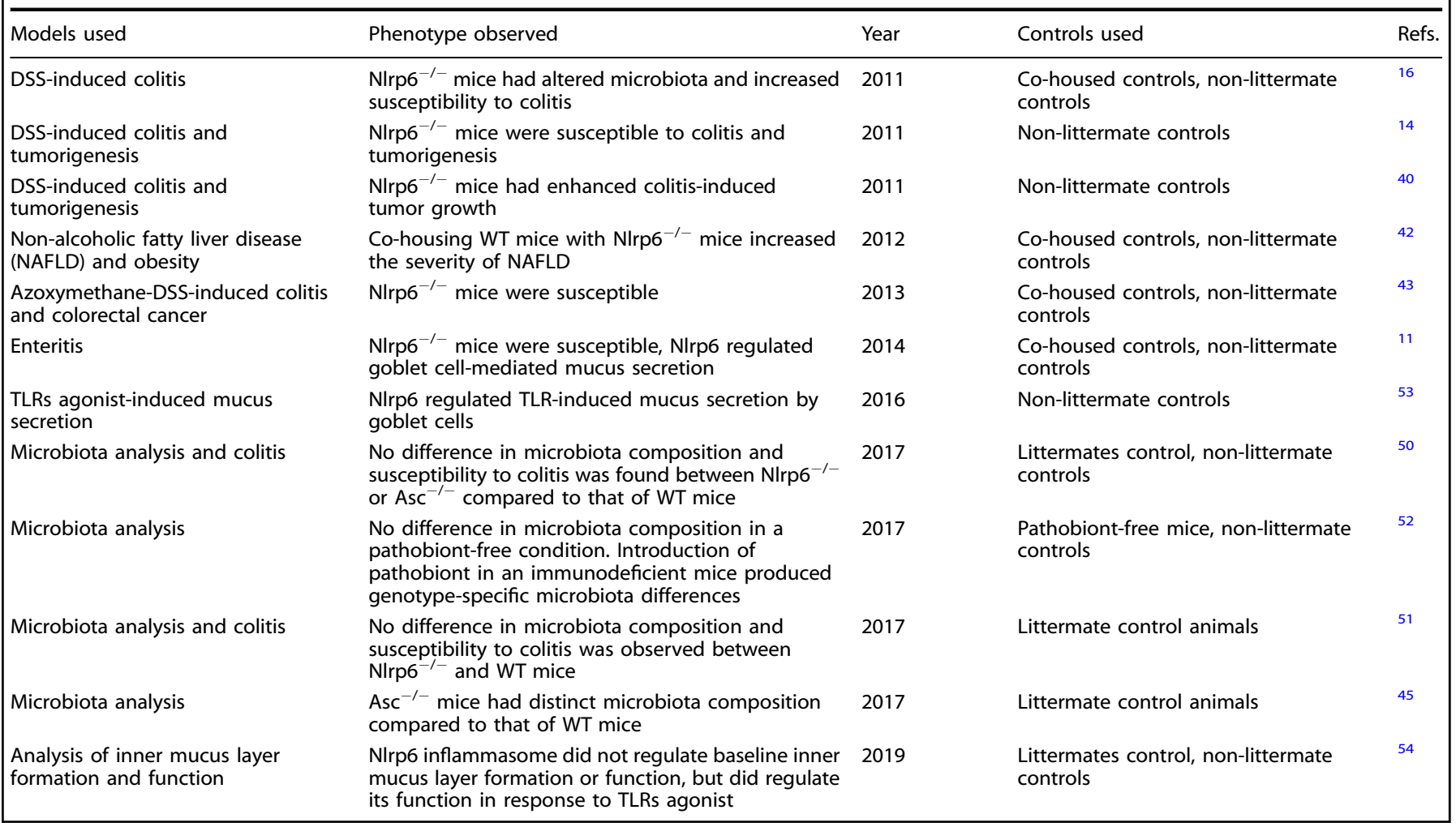

colonies of same genotype even within the same facility, suggesting that non-genetic factors (familial transmission and stochastic event) can influence microbiota. Moreover, the authors demonstrated that the presence of specific pathobiont within a facility could be attributed to genotype (Nlrp6 or Rag2)-associated differences in microbiota composition. Therefore, presence of a specific pathobiont in one facility but not in the other might contribute to discrepant results. Nonetheless, use of littermate control animals should be utilized over co-housing to normalize microbiota between different mice and to investigate microbiotadriven phenotypes. A list of some important mouse studies involved in microbiota-dependent phenotypes are listed in Table 2.

\section{NLRP6 IN EPITHELIAL INTEGRITY AND MICROBIOTA-EPITHELIAL CROSSTALK}

Two sheets of mucosal layers prevent direct contact of commensal microbes with the colonic epithelium. The outer mucosal layer is blanketed by muc2 mucin secreted by goblet cells and serves as a habitat for commensal microbiota. ${ }^{55-57}$ Breaching of the mucin layer leads to aberrant crypt morphology and can result in subsequent invasive adenocarcinoma and colorectal cancer. ${ }^{58}$ Interestingly, the Nlrp6 inflammasome has been linked to epithelial integrity through regulation of goblet cell function ${ }^{11,53}$ and secretion of antimicrobial peptides by enterocytes. ${ }^{17}$ Wlodarska et al. ${ }^{11}$ reported that Nlrp6 inflammasome signaling is essential for homeostatic mucin secretion by goblet cells. Nlrp6 ${ }^{-/-}$ mice demonstrated reduced autophagy and hyperplasia of goblet cells with a failure to exocytose mucin granules. As a result, these mice had only a thin mucus layer over the epithelium and increased susceptibility to enteric infections. However, a recent study by Volk et al. ${ }^{54}$ did not support the finding that Nlrp6 is essential for steady-state mucin secretion and inner mucus layer formation by goblet cells. The authors used numerous inflammasome knockout mice, including NIrp6 ${ }^{-/-}$and Nlrp6 $6^{+/+}$ littermates and combination of in vivo/ex vivo inner mucus layer analysis, to characterize the effect of Nlrp6 in inner mucus layer formation and barrier function and found no significant effect in any of these functions during steady state. The authors also found that IL-18 $18^{-/-}$mice had defective inner mucus layer; however, the phenotype was microbiota dependent and IL-18 independent because recombinant IL-18 did not restore inner mucus layer function. Using co-housing, the authors further showed that the Bacteroidales family S24-7 and genus Aldercrutzia covary with the inner mucus layer function, suggesting that these bacteria are associated with impenetrable inner mucus layer phenotype. In contrast, the authors did find that Nlrp6 is essential for sentinel goblet cell-mediated mucin secretion in response to Tlr1/2/4 ligands. ${ }^{54}$ The latter results confirm the study by Birchenough et al. ${ }^{53}$ who showed that microbial metabolites, such as LPS, lipid A, P3CSK4, and flagellin, but not LTA or bacterial DNA, are endocytosed by sentinel goblet cells and then induce intracellular reactive oxygen species (ROS).$^{53}$ These ROS further activate the Nlrp6 inflammasome to regulate compound exocytosis of goblet cells for muc2 secretion (Fig. 3). Overall, these studies suggest that Nlrp6 modulates goblet cell function and inner mucus layer formation only in response to optimal TLRs agonist and not in basal level. The discrepancy in the results obtained from these two different laboratories ${ }^{11,54}$ could be due to heavy reliance on histological staining in the study by Wlodarska et al. ${ }^{11} \mathrm{~A}$ combination of different techniques could help to generate better conclusions regarding functional studies of inner mucus layer.

Finally, Nlrp6 has also been shown to regulate antimicrobial peptide secretion to form a protective chemical barrier between microbiota and epithelium. ${ }^{17}$ For example, microbiota-derived metabolites such as Taurin acetate induced IL-18-mediated AMP secretion via activation of the Nlrp6 inflammasome (Fig. 3). However, a recent study by Volk et al. ${ }^{54}$ detected neither IL-18 receptor complex nor any biologically active form of IL-18 in 


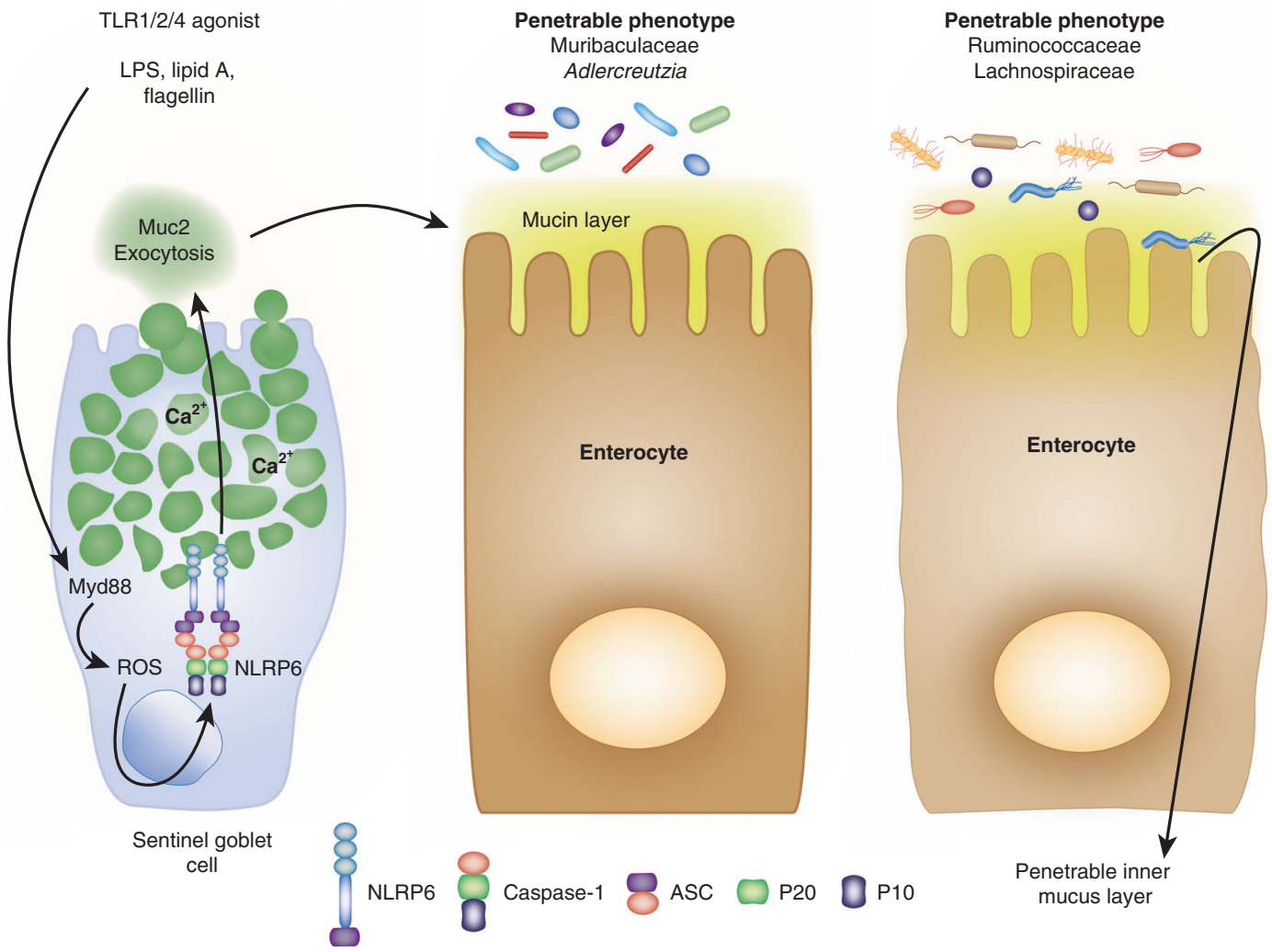

Fig. 3 The NLPR6 and microbiota-epithelium interface. Bacteria-derived TLR agonists such as LPS, lipid A, and flagellin activate the Myd88ROS pathway to activate the NLRP6 inflammasome in sentinel goblet cells. Activated NLRP6 facilitates the exocytosis of muc2 from goblet cells to form a physical barrier above the epithelium. In steady-state condition, microbiota such as Muribaculaceae and genus Adlercrutzia contribute to "impenetrable" inner mucus layer phenotype. The decrease in abundance of such microbiota could led to "penetrable" inner mucus layer characterized by a dysfunctional inner mucus layer. ROS: reactive oxygen species; LPS: lipopolysaccharides.

epithelium, which contradicts the proposed notion that epithelium-derived IL-18 regulates AMPs in a steady-state condition. Future studies will be important to shed light into this issue.

\section{NLRP6 IN METABOLIC DISEASES AND CARCINOGENESIS}

Studies using mouse models have demonstrated that Nlrp6 regulates the progression of NAFLD and obesity through regulation of gut microbiota. ${ }^{42}$ After feeding mice a methionine-choline-deficient diet for 4 weeks, Henao-Mejia et al. $^{42}$ found increased severity of NAFLD and non-alcoholic steatohepatitis (NASH) in inflammasome-deficient mice $\left(\mathrm{Asc}^{-/-}\right.$

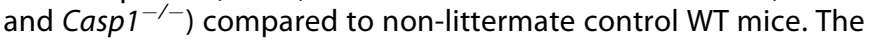
authors ascribed the difference to the production of IL-18 since $I L-18^{-/-}$, but not $I L-1 b^{-/-}$, mice displayed a phenotype similar to $\mathrm{Asc}^{-1-}$ and caspase $1^{-1-}$ mice. Moreover, co-housing WT mice with $\mathrm{Asc}^{-/-}, \mathrm{Casp}^{-/-}, \mathrm{IL} 18^{-/-}$, or Nlrp6 ${ }^{-/-}$mice enhanced the severity of NAFLD and NASH compared to singly housed WT mice, suggesting that the augmented susceptibility is mediated by differences in microbiota composition regulated through the Nlrp6-IL-18 signaling axis. Translocation of metabolites from the altered microflora in the gut of these mice via the portal vein activated TLR4 and TLR9 in the liver resulting in exacerbated NAFLD and NASH via tumor necrosis factor-a (TNF-a) secretion ${ }^{42}$ (Fig. 4). This study provides strong evidence that microbiota composition can dictate metabolic conditions, including dietinduced NAFLD and obesity. It will be however essential to investigate whether similar phenotype will be observed in littermate control animals since, as discussed above, recent studies using littermate control NIrp6 ${ }^{-/-}$and NIrp6 ${ }^{+/+}$mice displayed similar microbiota composition. Consistent with the finding from Henao-Mejia et al., ${ }^{42}$ a study by Lee et al. ${ }^{59}$ showed increased levels of IL-1 $\beta$ upon LPS treatment of mice fed a highfat diet suggesting involvement of inflammasomes in NAFLD. Moreover, treatment of hepatic cell lines (Hep G2) with palmitic acid and LPS resulted in the upregulation of the Nlrp6 inflammasome, an effect that was reversed by pretreatment with peroxisome proliferator-activated receptor- $\delta$ (PPAR- $\delta$ ) agonist, a nuclear receptor protein. However, this study did not reveal how PPAR- $\delta$ communicates with NIrp6 to induce inflammasome activation.

Another elegant study using a murine water avoidance stress (WAS) model reported that Nlrp6 inflammasome signaling is inhibited in WAS-induced enteritis via secretion of corticotrophinreleasing hormone $(\mathrm{CRH})$ from the brain as a result of stress. ${ }^{60}$ Further, this inhibition of Nlrp6 signaling led to dysbiosis-induced enteritis in stressed mice, which was transferrable to non-stressed mice upon co-housing. Interestingly, WAS-induced Nlrp6 inflammasome inhibition and associated intestinal pathology were reversed upon treatment with PPAR- $\gamma$ agonists. ${ }^{60}$ However, how $\mathrm{CRH}$ inhibits Nlrp6 inflammasome activation in the intestine remains unknown. It is possible that dysbiotic microflora observed in co-housed, non-stressed mice could affect the expression of the Nlrp6 inflammasome. Future studies are needed to shed light on these issues.

Nlrp6 deficiency has been implicated in the aggravation of chemical-induced colitis and subsequent tumorigenesis. ${ }^{14}$ Chen et al. ${ }^{14}$ induced chemical colitis in WT and Nlrp6 ${ }^{-1-}$ mice using DSS and found that NIrp6 ${ }^{-1-}$ mice had increased colitis-induced mortality and tumorigenesis compared to WT mice. However, recent studies ${ }^{50,51}$ using littermate control mice demonstrated no difference in susceptibility to colitis, suggesting again that 


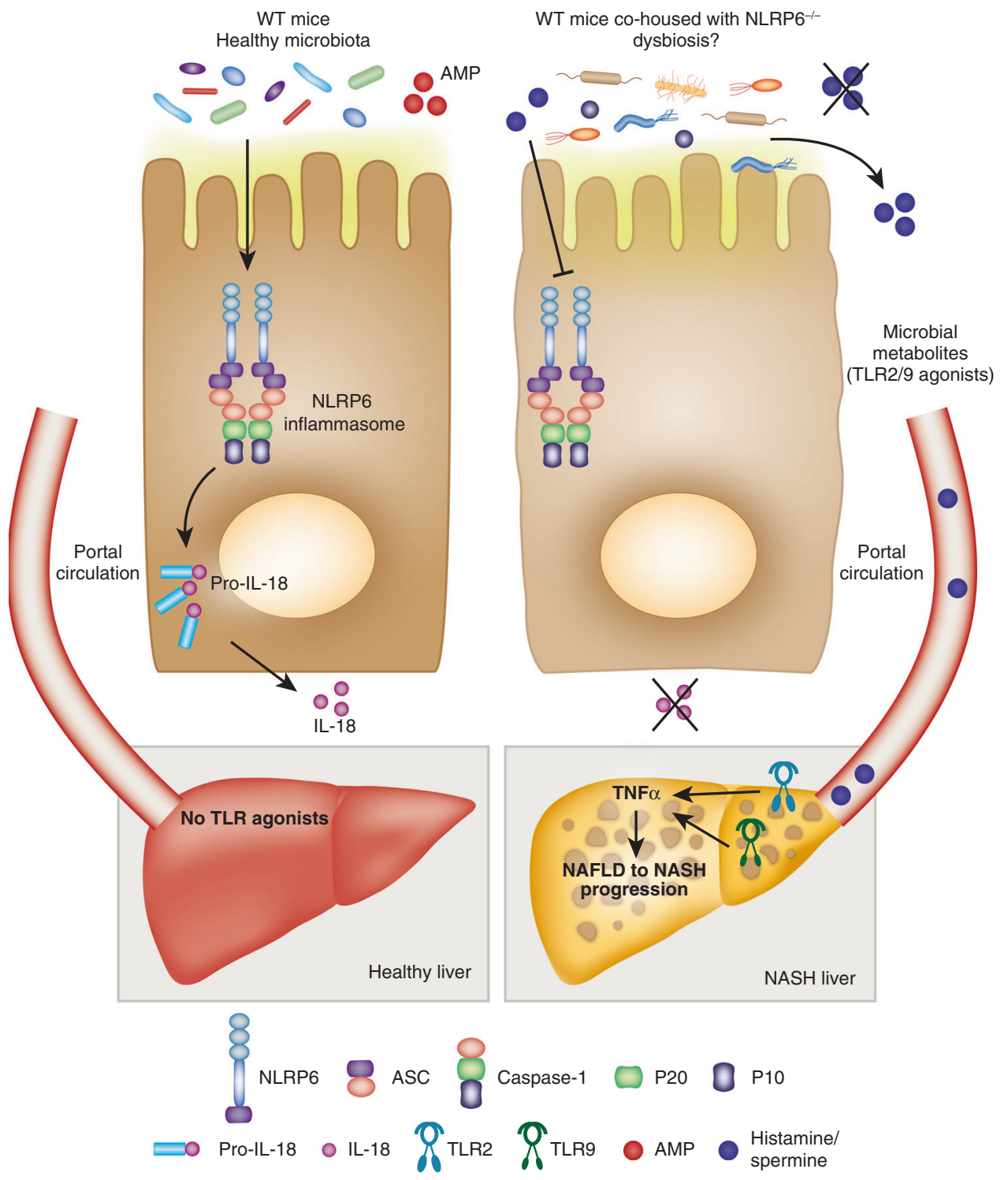

Fig. 4 NLRP6 in the gut-liver axis as observed in a study from non-littermate control mice. In a WT mouse, the NLRP6 inflammasome maintains a healthy epithelial cell-microbiota axis via AMP production by enterocytes. No abnormal metabolites are generated and hence the liver is not affected. When these mice are co-housed with NLRP6 ${ }^{-1-}$ mice, "dysbiotic" bacteria will outnumber normal healthy microbiota. TLR2 and TLR9 agonists secreted from "dysbiotic" microbiota reach the liver through the portal circulation where they activate TLR2 and TLR9, respectively, to induce TNF- $\alpha$ secretion. TNF- $\alpha$ will further enhance the progression of NAFLD to NASH. However, these phenotypes remained to be ascertained using littermate-controlled mice. AMP: antimicrobial peptide.

the increased susceptibility to colitis observed in NIrp6 ${ }^{-1-}$ mice could be due to the use of non-littermate controls. An interesting study by Normand et al. ${ }^{40}$ reported that Nlrp6 performs essential functions in the regulation of tissue repair necessary for protection against chemically induced colorectal carcinogenesis. This study showed that Nlrp6 deficiency led to dysregulated colonocyte proliferation and migration, thereby facilitating tumor formation. Reverse transcription-PCR analysis revealed increased expression of genes involved in cell proliferation such as casein kinase $\varepsilon(\operatorname{csnk} 1 \varepsilon)$ and SMARRC1 along with the proto-oncogene Myc11 in the DSS-treated colon of NIrp6 ${ }^{-1-}$ mice (Fig. 5). One year later, another well-designed study by $\mathrm{Hu}$ et al., ${ }^{43}$ using non-littermate control mice, suggested that the enhanced susceptibility to tumorigenesis in Nlrp6 $^{-1-}$ mice was microbiota dependent. The aberrant microflora in these mice caused increased transcription of CCL5, which, in turn, augmented epithelial proliferation via increased
IL-6 secretion (Fig. 5). However, additional studies using littermate controls will be essential to confirm these results. In another study, overexpression of NLRP6 in gastric cancer cells enhanced the expression of $\mathrm{P} 14^{\mathrm{ARF}}, \mathrm{P} 53$, and $\mathrm{P} 21$ while reducing the expression of Mdm2 and cyclin D1, suggesting that NLRP6 negatively regulates carcinogenesis via the $\mathrm{P} 14^{\mathrm{ARF}}-\mathrm{Mdm} 2-\mathrm{P} 53$ tumor suppressor axis ${ }^{61}$ (Fig. 5). Together, these observations establish Nlrp6 as a negative regulator of colorectal cancer and suggest that modulation of Nlrp6 may be a promising therapeutic alternative for its treatment.

\section{NLRPG IN NEUROINFLAMMATION}

In addition to its role in host defense against bacterial and viral infection-induced inflammation, NIrp6 has been shown to modulate neuroinflammation. ${ }^{15,62}$ In a murine model of acute peripheral nerve injury, Ydens et al. $^{15}$ reported that deletion of 


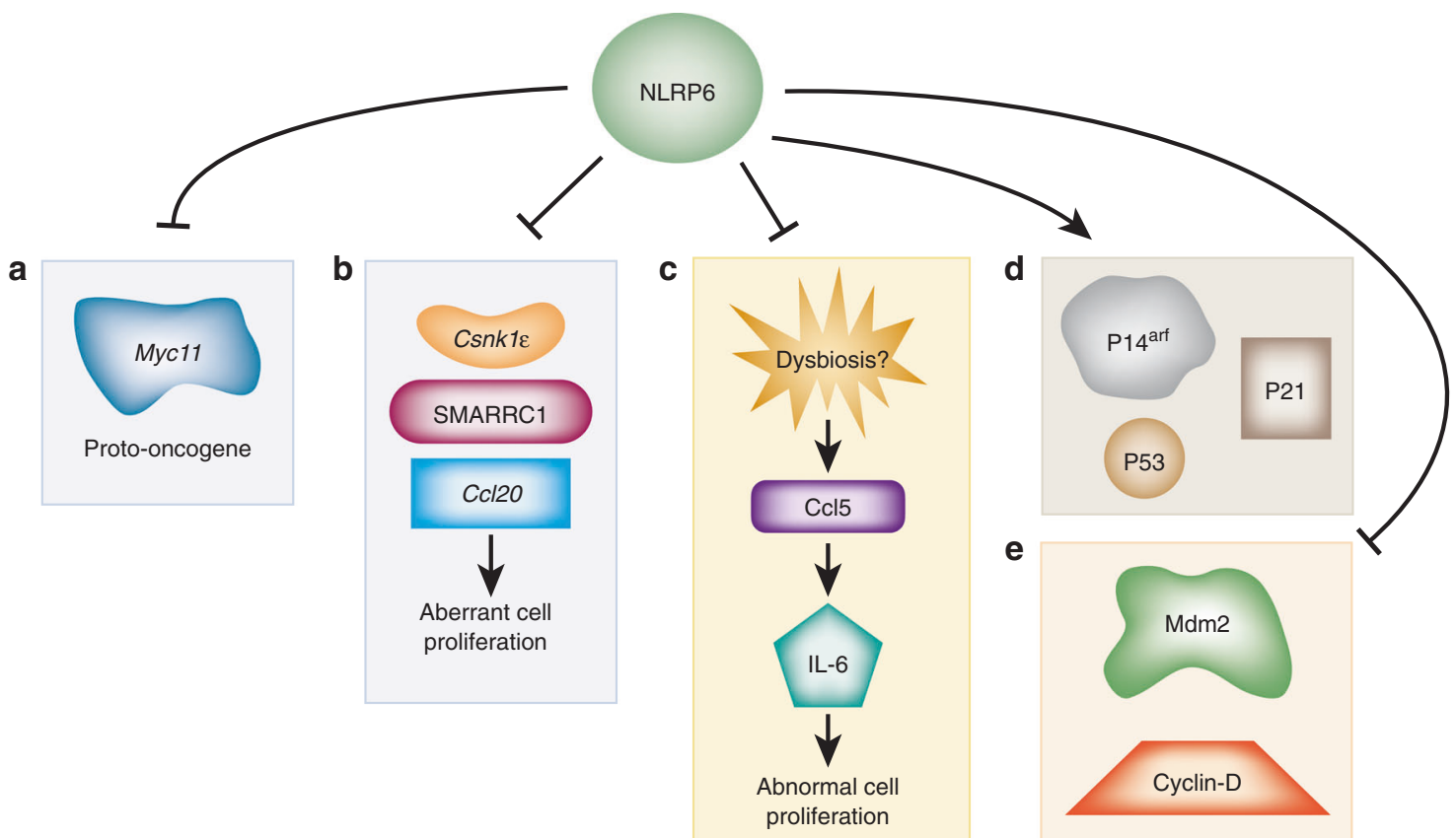

Fig. 5 NLRP6 modulates cellular mechanisms to prevent carcinogenesis. a, b NLRP6 deficiency increased the expression of genes involved in cell proliferation such as casein kinase $\varepsilon(\operatorname{csn} k 1 \varepsilon), c c / 20$, and SMARRC1 along with proto-oncogene Myc11 in the DSS-treated colon. c In a study using non-littermates control animals, NLRP6 prevented "dysbiosis"-induced IL-6 secretion, thereby preventing abnormal cell proliferation in the intestine. d, e NLRP6 augmented the expression of P14 ${ }^{\mathrm{ARF}}$, P21, and P53 and suppressed Mdm2 and cyclin D in gastric cancer cells to prevent tumor progression. Ccl20: CC-chemokine ligand 20; SMARRC1: a member of switch/sucrose nonfermentable chromatin remodeling complex; Ccl5: CC-chemokine ligand 5; Mdm2: murine double minute 2.

Nlrp6 strongly impaired nerve function. Further, the expression of both Nlrp6 and Nlrp3 was found to be increased $4 \mathrm{~h}$ after surgeryinduced acute neurodegeneration. Intriguingly, Nlrp6 ${ }^{-1-}$, but not $\mathrm{Nlrp3}^{-1-}$, mice had a dramatic drop in the sciatic functional index and needed a longer recovery time, compared with WT mice, after crushing of the right sciatic nerve. Furthermore, it was demonstrated that Nlrp6 enhanced this recovery independently of inflammasome activation. Thus, the authors proposed that enhanced MAPK activity observed in $\mathrm{Nlrp6}^{-/-}$mice could exacerbate inflammation resulting in delayed recovery from nerve injury. Future studies will be necessary to shed more light on how Nlrp6 enhances recovery from peripheral nerve injury and how this could be manipulated for therapeutic intervention.

In addition to the peripheral nervous system, a role for NIrp6 in the central nervous system has also been reported. In a murine model of intracerebral hemorrhage (ICH)-induced brain injury, Wang et al. ${ }^{62}$ analyzed the expression profiles and biological functions of Nlrp6. Nlrp6 expression was increased mainly in glial fibrillary acidic protein-positive astrocytes in the perihematomal brain after ICH downstream of TLR4 activation. Interestingly, $\mathrm{NIrp6}^{-/-}$mice displayed severe brain injury as evidenced by increased edema, NF-KB-mediated proinflammatory cytokine production, and aggravated neurological-deficit scores compared to their WT counterparts. Since microbiota has been shown to regulate neurodevelopmental disorders and promote the autism phenotype, ${ }^{63}$ it will be interesting to explore whether microbiota in the gut has any influence on $\mathrm{ICH}$-induced brain injury or in acute peripheral nerve injury.

\section{NLRP6 IN HUMAN DISEASES}

Most of the studies intended to define the expression and function of NLRP6 have been performed in mouse models and very little research to date has involved human patients. Consistent with the mouse model, genome-wide analysis based on gastrointestinal transcriptomics revealed high expression of NLRP6 in the small intestine of healthy humans, ${ }^{64}$ suggesting that NLRP6 might also have a specific role in the intestines of humans. Recently, we reported higher expression of NLRP6 in neutrophils, macrophages, and epithelial cells in the lungs obtained from pneumonia patients. MRSA-infected mice also displayed enhanced expression of Nlrp6 in these cells and a negative role of Nlrp6 in pulmonary host defense, ${ }^{12}$ suggesting that NLRP6 could have regulatory function in human lungs. While expression of Nlrp6 has been shown to prevent colorectal cancer in murine models, gene expression analysis performed in colorectal cancer patients showed no change in the expression of NLRP6. ${ }^{65}$ In contrast, a genome-wide study linked a single-nucleotide polymorphism in NLRP6 with platelet volume, ${ }^{66}$ suggesting that NLRP6 could have an important role in thrombopoiesis. Interestingly, the expression of NLRP6 and IL-1 $\beta$ was increased in adipose tissue obtained from portal fibrosis patients compared to that from control patients, ${ }^{67}$ suggesting a role of NLRP6 in the liver.

In another study of patients undergoing endodontic microsurgery, analysis of tissues associated with apical periodontitis revealed higher expression of NLRP6 ${ }^{68}$ In addition, when NLRP6 was silenced in periodontal ligament cells and then stimulated with E. coli-derived LPS, both NF-KB and ERK signaling were found to be augmented as were secretion of IL-6 and TNF-a. This suggests that NLRP6 suppresses the inflammatory response during periodontitis, which is in line with mouse studies where NLRP6 has been reported to negatively regulate NF-KB and ERK pathways. ${ }^{13}$ A similar anti-inflammatory role of NLRP6 has been reported in rheumatoid arthritis patients. In this study, NLRP6 was found to be downregulated in synovial tissues and fibroblast-like synoviocytes (FLS) at both the transcription and translational levels in rheumatoid arthritis patients compared to osteoarthritis patients. ${ }^{69}$ Silencing of NLRP6 in FLS from rheumatoid arthritis patients enhanced proinflammatory cytokine production via increased activity of NF-KB in response to TNF-a. Furthermore, 
the authors reported that NLRP6 acted as a docking site to facilitate the interaction between transforming growth factor- $\beta$ activated kinase- 1 binding protein $2 / 3$ and tripartite motif 38 to promote lysosome-dependent degradation. ${ }^{69}$ It is interesting that NLRP6 was found to regulate lysosome-mediated degradation, although future studies are needed to investigate if these findings have clinical relevance in other diseases.

\section{CONCLUDING REMARKS}

Nlrp6 was proposed to have multifaceted functions ranging from the regulation of host defense during microbial infections, composition of the epithelium-microbiota interface, and modulation of inflammatory modes of cell death such as pyroptosis and necroptosis, to regulation of metabolic diseases, neuroinflammation, and cancer. While some functions of Nlrp6 remain to be confirmed in the littermate control animals, the multitasking ability of Nlrp6 makes this inflammasome unique among NLR family members. Although NLRP6 was first reported to form an inflammasome in vitro in 2002, the long-standing conundrum regarding whether it can assemble an inflammasome in vivo or during microbial infection was only recently resolved. It is now known that Nlrp6 recognizes Listeria (LTA) and S. aureus and forms an inflammasome complex along with ASC to regulate host defense. Of interest is the finding that Nlrp6 recruits both caspase- 1 and caspase- 11 to form an inflammasome, whereas other NLR family members recruit only one or the other. Thus, future biophysical and biochemical studies will be essential to understand how caspase- 11 is recruited along with caspase-1 during Nlrp6 inflammasome assembly. Additionally, there likely remain many unknowns regarding this pathway as new inflammasomes, sensors, and downstream signaling pathways are constantly being elucidated.

Currently, there are large discrepancies regarding the role of Nlrp6 in the composition of gut microbiota. Future studies are essential to address this controversy. Moreover, a unanimous, comprehensive protocol should be established through a consortium in order to bring uniformity in microbiota research.

While most of the research studies regarding Nlrp6 have concentrated on the gut, the role of this molecule in host defense has also gained considerable attention. For example, a detrimental role of Nlrp6 in innate immunity of the lungs has recently been described. ${ }^{12}$ However, the function of this inflammasome in host defense during pathogen infections in other organs, including heart, brain, kidneys, and neurons, remains unexplored. In addition, it will be of interest to investigate how Nlrp6 regulates immunity during fungal infections and sepsis. Future studies in these areas will enhance our understanding of the role of Nlrp6 in host defense.

Several studies have suggested that the function of Nlrp6 is context dependent. While this molecule is important for limiting infections in the intestines, it appears to be detrimental to host defense in the lungs. During infections, expression of Nlrp6 in myeloid cells such as macrophages and neutrophils appears to be detrimental in the lungs; however, epithelial cells expressing NIrp6 might play protective roles in the intestine. Further investigation will be required to understand the cell- and organ-specific functions of Nlrp6. Studies involving deletion of Nlrp6 in specific cell compartments, such as myeloid cells, epithelial cells, or lymphocytes, could generate more conclusive findings. The future challenge will be to apply our current knowledge of inflammasome biology to attenuate the tissue injury induced by uncontrolled inflammation due to infectious and non-infectious diseases.

\section{ACKNOWLEDGEMENTS}

We would like to acknowledge members of the Center for Lung Biology and Diseases, including Tirumalai Rangasamy, Sivakumar Periasamy, Joseph DeCorte, John T. Le and Dinesh Bhattarai, for their helpful discussion. This work is supported by R01Al113720 (S.J.), R01HL091958 (S.J.), R01Al140500 (S.J.), R21Al133681 (S.J.), P20GM130555 (S.J.), and F31HL137287-01 (S.P.) grants and fellowships from the National Institute of Health.

\section{AUTHOR CONTRIBUTIONS}

L.G., S.P., and L.J. wrote the draft and S.J. edited the draft.

\section{ADDITIONAL INFORMATION}

Competing interests The authors declare no competing interests.

Publisher's note Springer Nature remains neutral with regard to jurisdictional claims in published maps and institutional affiliations.

\section{REFERENCES}

1. Takeuchi, O. \& Akira, S. Pattern recognition receptors and inflammation. Cell 140, 805-820 (2010).

2. Franchi, L., Warner, N., Viani, K. \& Nunez, G. Function of Nod-like receptors in microbial recognition and host defense. Immunol. Rev. 227, 106-128 (2009).

3. Schroder, K. \& Tschopp, J. The inflammasomes. Cell 140, 821-832 (2010).

4. Ravi Kumar, S. et al. Emerging roles of inflammasomes in acute pneumonia. Am. J. Respir. Crit. Care Med. 197, 160-171 (2018).

5. Man, S. M. \& Kanneganti, T. D. Regulation of inflammasome activation. Immunol. Rev. 265, 6-21 (2015)

6. Elliott, E. I. \& Sutterwala, F. S. Initiation and perpetuation of NLRP3 inflammasome activation and assembly. Immunol. Rev. 265, 35-52 (2015).

7. Leissinger, M., Kulkarni, R., Zemans, R. L., Downey, G. P. \& Jeyaseelan, S. Investigating the role of nucleotide-binding oligomerization domain-like receptors in bacterial lung infection. Am. J. Respir. Crit. Care Med. 189, 1461-1468 (2014).

8. Lamkanfi, M. \& Dixit, V. M. Mechanisms and functions of inflammasomes. Cell 157, 1013-1022 (2014).

9. Broz, P. \& Dixit, V. M. Inflammasomes: mechanism of assembly, regulation and signalling. Nat. Rev. Immunol. 16, 407-420 (2016).

10. Grenier, J. M. et al. Functional screening of five PYPAF family members identifies PYPAF5 as a novel regulator of NF-kappaB and caspase-1. FEBS Lett. 530, 73-78 (2002).

11. Wlodarska, M. et al. NLRP6 inflammasome orchestrates the colonic host-microbial interface by regulating goblet cell mucus secretion. Cell 156, 1045-1059 (2014).

12. Ghimire, L. et al. NLRP6 negatively regulates pulmonary host defense in Grampositive bacterial infection through modulating neutrophil recruitment and function. PLoS Pathog. 14, e1007308 (2018).

13. Anand, P. K. et al. NLRP6 negatively regulates innate immunity and host defence against bacterial pathogens. Nature 488, 389-393 (2012).

14. Chen, G. Y., Liu, M., Wang, F., Bertin, J. \& Nunez, G. A functional role for Nlrp6 in intestinal inflammation and tumorigenesis. J. Immunol. 186, 7187-7194 (2011).

15. Ydens, E. et al. Nlrp6 promotes recovery after peripheral nerve injury independently of inflammasomes. J. Neuroinflamm. 12, 143 (2015).

16. Elinav, E. et al. NLRP6 inflammasome regulates colonic microbial ecology and risk for colitis. Cell 145, 745-757 (2011).

17. Levy, M. et al. Microbiota-modulated metabolites shape the intestinal microenvironment by regulating NLRP6 inflammasome signaling. Cell 163, 1428-1443 (2015).

18. Hara, $H$. et al. The NLRP6 inflammasome recognizes lipoteichoic acid and reg ulates Gram-positive pathogen infection. Cell 175, 1651-1664 e1614 (2018).

19. Lu, A. et al. Unified polymerization mechanism for the assembly of ASCdependent inflammasomes. Cell 156, 1193-1206 (2014).

20. Park, Y. H., Wood, G., Kastner, D. L. \& Chae, J. J. Pyrin inflammasome activation and RhoA signaling in the autoinflammatory diseases FMF and HIDS. Nat. Immunol. 17, 914-921 (2016).

21. $\mathrm{Hu}, \mathrm{Z}$. et al. Crystal structure of NLRC4 reveals its autoinhibition mechanism Science 341, 172-175 (2013).

22. Cai, $X$. et al. Prion-like polymerization underlies signal transduction in antiviral immune defense and inflammasome activation. Cell 156, 1207-1222 (2014).

23. Lu, A. \& Wu, H. Structural mechanisms of inflammasome assembly. FEBS J. 282, 435-444 (2015)

24. Shi, J. et al. Cleavage of GSDMD by inflammatory caspases determines pyroptotic cell death. Nature 526, 660-665 (2015).

25. Shen, C. et al. Molecular mechanism for NLRP6 inflammasome assembly and activation. Proc. Natl Acad. Sci. USA 116, 2052-2057 (2019). 
26. Lu, A. et al. Plasticity in PYD assembly revealed by cryo-EM structure of the PYD filament of AIM2. Cell Discov. 1, 15013 (2015).

27. Diebolder, C. A., Halff, E. F., Koster, A. J., Huizinga, E. G. \& Koning, R. I. Cryoelectron tomography of the NAIP5/NLRC4 inflammasome: implications for NLR activation. Structure 23, 2349-2357 (2015).

28. Paudel, S. et al. NLRC4 suppresses IL-17A-mediated neutrophil-dependent host defense through upregulation of IL-18 and induction of necroptosis during Gram-positive pneumonia. Mucosal Immunol. 12, 247-257 (2019).

29. Strowig, T., Henao-Mejia, J., Elinav, E. \& Flavell, R. Inflammasomes in health and disease. Nature 481, 278-286 (2012)

30. Broz, P. \& Monack, D. M. Molecular mechanisms of inflammasome activation during microbial infections. Immunol. Rev. 243, 174-190 (2011).

31. Gross, O. et al. Syk kinase signalling couples to the Nlrp3 inflammasome for antifungal host defence. Nature 459, 433-436 (2009).

32. Kebaier, C. et al. Staphylococcus aureus alpha-hemolysin mediates virulence in a murine model of severe pneumonia through activation of the NLRP3 inflammasome. J. Infect. Dis. 205, 807-817 (2012).

33. Mangan, M. S. J. et al. Targeting the NLRP3 inflammasome in inflammatory diseases. Nat. Rev. Drug Discov. 17, 588-606 (2018).

34. Wang, W. et al. Zika virus infection induces host inflammatory responses by facilitating NLRP3 inflammasome assembly and interleukin-1 beta secretion. Nat. Commun. 9, 106 (2018).

35. Rimessi, A. et al. Mitochondrial $\mathrm{Ca}^{2+}$-dependent NLRP3 activation exacerbates the Pseudomonas aeruginosa-driven inflammatory response in cystic fibrosis. Nat. Commun. 6, 6201 (2015).

36. Wang, P. et al. Nlrp6 regulates intestinal antiviral innate immunity. Science $\mathbf{3 5 0}$, 826-830 (2015).

37. Kitur, K. et al. Toxin-induced necroptosis is a major mechanism of Staphylococcus aureus lung damage. PLoS Pathog. 11, e1004820 (2015).

38. Jorgensen, I. \& Miao, E. A. Pyroptotic cell death defends against intracellular pathogens. Immunol. Rev. 265, 130-142 (2015).

39. Toubai, T. et al. Host NLRP6 exacerbates graft-versus-host disease independent of gut microbial composition. Nat. Microbiol. 4, 800-812 (2019).

40. Normand, S. et al. Nod-like receptor pyrin domain-containing protein 6 (NLRP6) controls epithelial self-renewal and colorectal carcinogenesis upon injury. Proc. Natl Acad. Sci. USA 108, 9601-9606 (2011).

41. Wang, Y. et al. The intestinal microbiota regulates body composition through NFIL3 and the circadian clock. Science 357, 912-916 (2017).

42. Henao-Mejia, J. et al. Inflammasome-mediated dysbiosis regulates progression of NAFLD and obesity. Nature 482, 179-185 (2012).

43. Hu, B. et al. Microbiota-induced activation of epithelial IL-6 signaling links inflammasome-driven inflammation with transmissible cancer. Proc. Natl Acad. Sci. USA 110, 9862-9867 (2013).

44. Seregin, S. S. et al. NLRP6 protects II10(-/-) mice from colitis by limiting colonization of Akkermansia muciniphila. Cell Rep. 19, 733-745 (2017).

45. Levy, M., Shapiro, H., Thaiss, C. A. \& Elinav, E. NLRP6: a multifaceted innate immune sensor. Trends Immunol. 38, 248-260 (2017).

46. Rausch, P. et al. Analysis of factors contributing to variation in the C57BL/6J fecal microbiota across German animal facilities. Int. J. Med. Microbiol. 306, 343-355 (2016).

47. Ubeda, C. et al. Familial transmission rather than defective innate immunity shapes the distinct intestinal microbiota of TLR-deficient mice. J. Exp. Med. 209, 1445-1456 (2012).

48. Turnbaugh, P. J. et al. The effect of diet on the human gut microbiome: a metagenomic analysis in humanized gnotobiotic mice. Sci. Transl. Med. 1, 6ra14 (2009).
49. Robertson, S. J. et al. Comparison of co-housing and littermate methods for microbiota standardization in mouse models. Cell Rep. 27, 1910-1919 e1912 (2019).

50. Mamantopoulos, M. et al. Nlrp6- and ASC-dependent inflammasomes do not shape the commensal gut microbiota composition. Immunity 47, 339-348 e334 (2017).

51. Lemire, P. et al. The NLR protein NLRP6 does not impact gut microbiota composition. Cell Rep. 21, 3653-3661 (2017).

52. Galvez, E. J. C., Iljazovic, A., Gronow, A., Flavell, R. \& Strowig, T. Shaping of intestinal microbiota in Nlrp6- and Rag2-deficient mice depends on community structure. Cell Rep. 21, 3914-3926 (2017).

53. Birchenough, G. M., Nystrom, E. E., Johansson, M. E. \& Hansson, G. C. A sentinel goblet cell guards the colonic crypt by triggering Nlrp6-dependent Muc2 secretion. Science 352, 1535-1542 (2016).

54. Volk, J. K. et al. The Nlrp6 inflammasome is not required for baseline colonic inner mucus layer formation or function. J. Exp. Med. 216, 2602-2618 (2019).

55. Johansson, M. E., Larsson, J. M. \& Hansson, G. C. The two mucus layers of colon are organized by the MUC2 mucin, whereas the outer layer is a legislator of host-microbial interactions. Proc. Natl Acad. Sci. USA 108(Suppl. 1), 4659-4665 (2011).

56. Tytgat, K. M. et al. Biosynthesis of human colonic mucin: Muc2 is the prominent secretory mucin. Gastroenterology 107, 1352-1363 (1994).

57. Pelaseyed, T. et al. The mucus and mucins of the goblet cells and enterocytes provide the first defense line of the gastrointestinal tract and interact with the immune system. Immunol. Rev. 260, 8-20 (2014).

58. Velcich, A. et al. Colorectal cancer in mice genetically deficient in the mucin Muc2. Science 295, 1726-1729 (2002).

59. Lee, H. J. et al. Peroxisome proliferator-activated receptor-delta agonist ameliorated inflammasome activation in nonalcoholic fatty liver disease. World J. Gastroenterol. 21, 12787-12799 (2015).

60. Sun, Y. et al. Stress-induced corticotropin-releasing hormone-mediated NLRP6 inflammasome inhibition and transmissible enteritis in mice. Gastroenterology 144, 1478-1487 (2013). 1487 and 1471-1478

61. Wang, H. et al. Correction: NLRP6 targeting suppresses gastric tumorigenesis via P14(ARF)-Mdm2-P53-dependent cellular senescence. Oncotarget 9, 35512 (2018).

62. Wang, P. F. et al. NLRP6 inflammasome ameliorates brain injury after intracerebral hemorrhage. Front. Cell Neurosci. 11, 206 (2017).

63. Kim, S. et al. Maternal gut bacteria promote neurodevelopmental abnormalities in mouse offspring. Nature 549, 528-532 (2017).

64. Gremel, G. et al. The human gastrointestinal tract-specific transcriptome and proteome as defined by RNA sequencing and antibody-based profiling. J. Gastroenterol. 50, 46-57 (2015).

65. Liu, R. et al. Expression profile of innate immune receptors, NLRs and AIM2, in human colorectal cancer: correlation with cancer stages and inflammasome components. Oncotarget 6, 33456-33469 (2015).

66. Gieger, C. et al. New gene functions in megakaryopoiesis and platelet formation. Nature 480, 201-208 (2011).

67. Mehta, R. et al. Expression of NALPs in adipose and the fibrotic progression of non-alcoholic fatty liver disease in obese subjects. BMC Gastroenterol. 14, 208 (2014).

68. Lu, W. L. et al. NLRP6 suppresses the inflammatory response of human periodontal ligament cells by inhibiting NF-kappaB and ERK signal pathways. Int. Endod. J. 52, 999-1009 (2019).

69. Lin, Y. \& Luo, Z. NLRP6 facilitates the interaction between TAB2/3 and TRIM38 in rheumatoid arthritis fibroblast-like synoviocytes. FEBS Lett. 591, 1141-1149 (2017). 